\title{
Checklist of helminth parasites in wild carnivore mammals from Brazil
}

\author{
FABIANO M. VIEIRA ${ }^{1,4}$, JOSÉ L. LUQUE ${ }^{2} \&$ LUÍS C. MUNIZ-PEREIRA ${ }^{3}$ \\ ${ }^{1}$ Laboratório de Taxonomia e Ecologia de Helmintos, Departamento de Zoologia, Universidade Federal de Juiz de Fora, Campus \\ Universitário, Juiz de Fora, MG, Brazil, CEP 36036-330. E-mail: fmatosvieira@gmail.com \\ ${ }^{2}$ Departamento de Parasitologia Animal, Universidade Federal Rural do Rio de Janeiro, Caixa Postal 74.508, Seropédica, RJ, Brazil, \\ CEP 23851-970.E-mail: jlluque@ufrrj.br \\ ${ }^{3}$ Laboratório de Helmintos Parasitos de Vertebrados, Instituto Oswaldo Cruz, FIOCRUZ, Av. Brasil 4365, RJ, Brazil, CEP $21040-900$. \\ E-mail:Imuniz@ioc.fiocruz.br \\ ${ }^{4}$ Pós-Gradução em Biologica Animal, Instituto de Biologia, Universidade Federal Rural do Rio de Janeiro, Seropédica, RJ, Brazil
}

\begin{abstract}
Ninety-five helminth parasite species totaling 480 records (including 60 new host and geographical records) in 21 species of wild carnivore mammals from Brazil were listed. Nineteen undetermined helminth species and 4 undetermined host species were also included. Information about the site of infection of parasites, localities, references and a host-parasite list were included herein.
\end{abstract}

Key words: Helminths, Biodiversity, wild carnivore mammals, Neotropics, Brazil

\section{Introduction}

Brazil is the fifth largest country in the world, and the first of the megadiversity countries, accounting for roughly $14 \%$ of the world's biota (Lewinsohn \& Prado 2002). It has the largest mammal diversity (more than 530 described species) with many yet to be discovered and catalogued (Costa et al. 2005). Although mammal conservation had benefited from a number of recent initiatives by the government and nongovernmental organizations, carnivores is one of the mammals group most threatened in Brazil (Costa et al. 2005).

The parasites are recognized as an important component of global biodiversity and the research effort directed to documenting parasite species have been increased on the last years (Poulin \& Morand 2004). With an increasing awareness by the general public and by the scientific community in function of the accelerating loss of biological diversity, comparative tests using parasites as indicators of ecological age and diversity will come as more complete studies of biotic diversity in different geographic areas on earth become available reinforcing the needs for more information on parasite fauna (Gardner \& Campbell 1992).

Some previous monographs (Yamaguti 1959, 1963, 1971; Travassos 1965; Travassos et al. 1969; Schmidt 1986; Thatcher 1993; and Vicente et al. 1997) included records of helminth parasites of Brazilian carnivore mammals inside on generalized compilations. Here we present the first effort to compile and summarize the helminth parasites of wild carnivore mammals from Brazil. This is also the first attempt to include massive information from the Helminthological Collection of the Oswaldo Cruz Institute (CHIOC), Rio de Janeiro, Brazil, which is unpublished. 


\section{Material and methods}

Using published records and unpublished information retrieved from the Helminthological Collection of the Oswaldo Cruz Institute (CHIOC), a checklist of the helminth parasites of carnivore mammals from Brazil was generated. In addition to published records, a total of 892 parasite specimens samples stored in the CHIOC from 1901 to 1996, were examined. The checklist follows the classification and systematic arrangement of Gibson et al. (2002) and Jones et al. (2005) for Digenea, Khalil et al. (1994) for Cestoda, Chabaud (1975, 1978), Anderson \& Bain (1976, 1982), Lichtenfels (1980a, b), Durette-Desset (1983) and Vicente et al. (1997) for Nematoda, Schmidt (1972) and Amin (1985, 1987) for Acanthocephala.

The species of helminths are presented in alphabetical order, followed by hosts (specific name), site of infection, localities and references (between parentheses, in chronological sequence). In addition, the checklist included helminth species recorded only to genus level (undetermined species). Helminth species names follow the most recent taxonomic literature. Mention of the helminths and host species in this list does not imply that the authors agree with their validity or taxonomy. Higher-level classification of mammals follows Wozencraft (2005). Species of hosts are arranged in alphabetical sequence. The following conventions in relation to the parasite records are observed: NHR refers to a new host record in Brazil and NGR to a new geographical record. Voucher specimens of the new parasitological records are deposited in the CHIOC. The CHIOC catalog number was included for all species with type specimens and voucher specimens deposited in the Collection.

\section{Results}

Ninety-five helminth parasite species totalling 480 records (including 60 new host and geographical records) in 21 species of wild carnivore mammals from Brazil were listed. Nineteen undetermined helminth species and four undetermined host species were also included. The most cited species was Dioctophyma renale with 16 records. The host with the most diverse parasite fauna was Cerdocyon thous (Linnaeus) with 24 helminth species.

\section{Phylum Acanthocephala Rudolphi, 1809}

Class Archiacanthocephala Meyer, 1931

\section{Order Oligacanthorhynchida Petrochenko, 1956}

\section{Family Oligacanthorhynchidae Southwell \& Macfie, 1925}

Neoncicola pintoi (Machado Filho, 1950)

Conepatus chinga (Molina), unspecified site of infection, Rio Grande do Sul (Machado Filho 1950)

(CHIOC 7730-paratype, 17824—allotype, 17825-17826-paratypes, 17850-holotype, 17851paratype).

Neoncicola potosi (Machado Filho, 1950)

Potos flavus (Schreber), large intestine, Pará (Machado Filho 1950) (CHIOC 10592—paratype,

17829-holotype and allotype, 17830 — paratype).

Oligacanthorhynchus decrescens (Meyer, 1931)

Leopardus wiedii (Schinz), unspecified site of infection, unspecified locality (Yamaguti 1963).

Oligacanthorhynchus pardalis (Westrumb, 1821) 
Leopardus pardalis (Linnaeus), small intestine, São Paulo, Mato Grosso do Sul (Travassos 1917) (CHIOC 1452, 16176).

Leopardus (Oncifelis) geoffroyi (D’Orbigny \& Gervais), intestine, Rio Grande do Sul, São Paulo (Travassos 1917) (CHIOC 1454).

Panthera onca (Linnaeus), intestine, unspecified locality (Travassos 1917).

Puma concolor (Linnaeus), intestine, unspecified locality (Travassos 1917).

Oncicola campanulata (Diesing, 1851)

Leopardus pardalis (Linnaeus), unspecified site of infection, unspecified locality (Meyer 1931; Yamaguti 1963; Machado Filho 1964)

Leopardus (Oncifelis) geoffroyi (D’Orbigny \& Gervais), unspecified site of infection, unspecified locality (Meyer 1931; Machado Filho 1964).

Panthera onca (Linnaeus), unspecified site of infection, unspecified locality (Meyer 1931; Machado Filho 1964).

Puma concolor (Linnaeus), unspecified site of infection, unspecified locality (Meyer 1931; Machado Filho 1964).

Oncicola chibigouzouensis Machado Filho, 1963

Leopardus pardalis (Linnaeus), small intestine, Mato Grosso (Machado Filho 1963a; Marteu 1977)

(CHIOC 3987, 3988, 28769—allotype, 28770 - 28778-paratypes).

Oncicola luehei (Travassos, 1917)

Nasua nasua (Linnaeus), intestine, large intestine, Pará, São Paulo, Minas Gerais, Mato Grosso, Mato

Grosso do Sul (Travassos 1917; Travassos et al. 1927; Lent \& Freitas 1938a; Machado Filho 1940;

Machado Filho 1950) (CHIOC 437, 438, 1418, 1419, 1431, 1432, 1439, 2371, 2534, 4026, 4027 -

4029, 7552, 9179, 9969, 9970, 10573, 11059, 13569, 16181-16183, 17823; 17856-lectoholotype, 17857-lectoallotype, 18046).

Oncicola macrurae Meyer, 1931

Leopardus wiedii (Schinz), small intestine, intestine, Pará (Lent \& Freitas 1938a, Marteu 1977;

Machado Filho 1964) (CHIOC 10145, 10576, 13568, 29022 - 29025, 29028 - 29030).

Oncicola magalhaesi Machado Filho, 1962

Puma concolor (Linnaeus), small intestine, São Paulo (Machado Filho 1962) (CHIOC 28785-holotype, 28786-allotype, 28787- 28801-paratypes).

Oncicola micracantha Machado Filho, 1949

Conepatus chinga (Molina), small intestine, Rio Grande do Sul (Machado Filho 1964; Marteu 1977) (CHIOC 17729).

Oncicola oncicola (Ihering, 1892)

Panthera onca (Linnaeus), stomach, intestine, small intestine, São Paulo, Minas Gerais (Travassos 1917; Machado Filho 1964) (CHIOC No 461, 1433-paratype, 1434, 1437, 1438, 4412, 6319, 7187, 28983 - 28988, 29006 - 29021, 29228 - 29235).

Puma (Herpailurus) yagouaroundi (Geoffroy), stomach, intestine, unspecified locality (Travassos 1917).

Leopardus pardalis (Linnaeus), stomach, intestine, São Paulo (Travassos 1917).

Leopardus wiedii (Schinz), small intestine, Pará, NHR (CHIOC 29026, 29027, 29031).

Oncicola paracampanulata Machado Filho, 1963

Puma (Herpailurus) yagouaroundi (Geoffroy), small intestine, São Paulo, Pará, Paraná (Machado

Filho 1963b; Machado Filho 1964; Marteu 1977) (CHIOC 462, 796, 813-paratype, 21362paratype, 28779-holotype, 28780—allotype, 29781 - 28784—paratypes).

Oncicola sigmoides (Meyer, 1932)

Galictis sp., unspecified site of infection, unspecified locality (Yamaguti 1963). 
Conepatus sp., unspecified site of infection, unspecified locality (Yamaguti 1963).

Oncicola sp.

Cerdocyon thous (Linnaeus), large intestine, under the skin, Espírito Santo, Mato Grosso do Sul, NHR (CHIOC 16185, 28095).

Leopardus pardalis (Linnaeus), large intestine, small intestine, Mato Grosso, São Paulo (Travassos 1945) (CHIOC 15715).

Pachysentis gethi (Machado Filho, 1950)

Galictis cuja (Molina), large intestine, Rio de Janeiro, NHR (CHIOC 9203-paratype, 17838paratype).

Eira barbara (Linnaeus), small intestine, large intestine, Pará, Rio de Janeiro (Machado Filho 1950)

(CHIOC 15680-paratype, 17836-holotype and allotype, 17837-paratype, 17841-paratype, 17846-paratype, 17852-paratype).

Galictis vittata (Schreber), large intestine, Rio de Janeiro, NHR (CHIOC 4144).

Pachysentis procyonis (Machado Filho, 1950)

Procyon cancrivorus (G. Cuvier), large intestine, small intestine, Rio de Janeiro (Machado Filho 1950) (CHIOC 17831- allotype, 17832-holotype, 17833-paratype, 17847-paratype, 17854paratype, 27002).

Prosthenorchis elegans (Diesing, 1851)

Eira barbara (Linnaeus), intestine, Rio de Janeiro (Travassos 1917).

Conepatus chinga (Molina), intestine, Piaú (Travassos 1917) (CHIOC 441, 804).

Prosthenorchis sp.

Leopardus pardalis (Linnaeus), intestine, Pará, NHR (CHIOC 10123).

\section{Class Palaeacanthocephala Meyer, 1931}

Order Polymorphida Petrochenko, 1956

Family Polymorphidae Meyer, 1931

Hexaglandula mutabilis (Rudolphi, 1819)

Lontra sp., large intestine, Rio de Janeiro, NHR (CHIOC 5399).

\section{Phylum Nematoda (Rudolphi, 1808)}

\section{Superfamily Acuarioidea Molin, 1860}

\section{Family Acuariidae Seurat, 1913}

Skrjabinoclava thapari Freitas, 1935

Procyon cancrivorus (G. Cuvier), stomach, Rio de Janeiro (Vicente et al. 1997) (CHIOC 18135holotype, 18136 - allotype, 18137-paratype, 1205).

\section{Superfamily Ancylostomatoidea Chabaud, 1965}

Family Ancylostomatidae (Looss, 1905)

Ancylostoma bidens (Molin, 1861)

Nasua nasua (Linnaeus), intestine, unspecified locality (Vicente et al. 1997).

Procyon cancrivorus (G. Cuvier), intestine, unspecified locality (Vicente et al. 1997). 
Puma (Herpailurus) yagouaroundi (Geoffroy), body cavity, intestine, Pará, Mato Grosso do Sul (Noronha et al. 2002).

Ancylostoma braziliense Faria, 1910

Leopardus pardalis (Linnaeus), intestine, Pará (Noronha et al. 2002) (CHIOC 10128).

Procyon cancrivorus (G. Cuvier), small intestine, Rio de Janeiro (Vicente et al. 1997) (CHIOC 15423).

Puma concolor (Linnaeus), stomach, small intestine, Rio de Janeiro, Paraná (Noronha et al. 2002) (CHIOC 4258, 19804).

Puma (Herpailurus) yaguarondi (Geoffroy), abdominal cavity, intestine, Pará, NHR (CHIOC 15061, 16070, 21363 - 21367).

Ancylostoma buckleyi Le Roux \& Biocca, 1957

Cerdocyon thous (Linnaeus), small intestine, São Paulo (Santos et al. 2003).

Ancylostoma caninum (Ercolani, 1859)

Cerdocyon thous (Linnaeus), intestine, small intestine, stomach, São Paulo, Rio de Janeiro, Mato Grosso do Sul, Pará (Noronha et al. 2004) (CHIOC 1738, 3073, 8298, 13579, 15156, 15159, 15164, 18338, 18923, 33808).

Chrysocyon brachyurus (Illiger), unspecified site of infection, Minas Gerais (Costa \& Freitas 1967; Mundim et al. 1991; Vicente et al. 1997).

Panthera onca (Linnaeus), unspecified site of infection, Mato Grosso, NHR (CHIOC 255).

Lycalopex gymnocercus (G. Fischer), small intestine, Mato Grosso do Sul, Rio de Janeiro, NHR (CHIOC 13063).

Lycalopex sp., small intestine, Rio de Janeiro, NHR (CHIOC 3067).

Puma (Herpailurus) yagouaroundi (Geoffroy), intestine, Mato Grosso, NHR (CHIOC 8855).

Ancylostoma conepati Solanet, 1911

Conepatus chinga (Molina), small intestine, Rio Grande do Sul, NGR (CHIOC 13970, 13971, 13973, 14003).

Ancylostoma pluridentatum Alessandri, 1905

Leopardus wiedii (Schinz), small intestine, Pará, NGR (CHIOC 9041).

Panthera onca (Linnaeus), small intestine, Amazonas, NGR (CHIOC 29137).

uma concolor (Linnaeus), small intestine, Rio de Janeiro, NGR (CHIOC 8997, 8959).

Necator americanus (Stiles, 1902)

Potos flavus (Schreber), intestine, Pará, NHR (CHIOC 9867).

Necator urichi Cameron, 1936

Procyon cancrivorus (G. Cuvier), small intestine, Rio de Janeiro (Vicente et al. 1997) (CHIOC 18133

- allotype, 18134, 18204).

Uncinaria bidens Lent \& Freitas, 1938

Nasua nasua (Linnaeus), intestine, unspecified locality (Vicente et al. 1997).

Procyon cancrivorus (G. Cuvier), intestine, unspecified locality (Vicente et al. 1997).

Uncinaria carinii Travassos, 1915

Cerdocyon thous (Linnaeus), instestine, São Paulo (Travassos, 1915, Vicente et al. 1997) (CHIOC 6368).

Uncinaria maxillaris (Molin, 1861)

Procyon cancrivorus (G. Cuvier), intestine, São Paulo (Vicente et al. 1997).

Uncinaria stenocephala (Railliet, 1884)

Chrysocyon brachyurus (Illiger), small intestine, Minas Gerais (Mundim et al. 1991).

Uncinaria sp.

Chrysocyon brachyurus (Illiger), intestine, Minas Gerais (Costa \& Freitas 1967; Vicente et al. 1997). 


\section{Superfamily Ascaridoidea Railliet \& Henry, 1915}

\section{Family Ascarididae Baird, 1853}

Toxascaris leonina (Linstow, 1902)

Eira barbara (Linnaeus), small intestine, Mato Grosso do Sul (Noronha et al. 2002) (CHIOC 14566). Leopardus pardalis (Linnaeus), small intestine, stomach, Paraná (Vicente et al. 1997) (CHIOC 33814).

Panthera onca (Linnaeus), small intestine, stomach, Mato Grosso (Vicente et al. 1997) (CHIOC 19492).

Puma concolor (Linnaeus), small intestine, stomach, Rio de Janeiro, Paraná (Noronha et al. 2002) (CHIOC 4208, 8994, 8996, 33813).

Puma (Herpailurus) yagouaroundi (Geoffroy), stomach, Paraná, NHR (CHIOC 33815).

Toxascaris sp.

Eira barbara (Linnaeus), intestine, Mato Grosso, NHR (CHIOC 14563).

Toxocara alienata (Rudolphi, 1819)

Nasua nasua (Linnaeus), small intestine, intestine, Minas Gerais, Pará (Vicente et al. 1997) (CHIOC 8748, 9471).

Procyon cancrivorus (G. Cuvier), intestine, unspecified locality (Vicente et al. 1997).

Toxocara canis (Werner, 1782)

Cerdocyon thous (Linnaeus), small intestine, Rio de Janeiro (Noronha et al., 2002).

Leopardus pardalis (Linnaeus), small intestine, Pará (Noronha et al. 2002) (CHIOC 21391).

Nasua nasua (Linnaeus), small intestine, stomach, Paraná, NHR (CHIOC 33810).

Procyon cancrivorus (G. Cuvier), esophagus, stomach, intestine, Mato Grosso, Rio de Janeiro, NHR (CHIOC 8097, 8203, 13678).

Puma concolor (Linnaeus), small intestine, Rio de Janeiro (Noronha et al. 2002).

Toxocara mistax (Zeder, 1800)

Puma (Herpailurus) yagouaroundi (Geoffroy), small intestine, Mato Grosso do Sul (Noronha et al., 2002) (CHIOC 15049).

Panthera onca (Linnaeus), unspecified site of infection, Mato Grosso (Noronha et al. 2002) (CHIOC 1779, 15706 - 15708).

Puma concolor (Linnaeus), stomach, São Paulo (Noronha et al. 2002) (CHIOC 27271).

Leopardus pardalis (Linnaeus), stomach, large intestine, small intestine, São Paulo, Mato Grosso, Amazonas, Pará, NHR (CHIOC 10086, 15716, 15718, 21215).

Toxocara sp.

Leopardus pardalis (Linnaeus), small intestine, Mato Grosso (Noronha et al. 2002) (CHIOC 8115).

Family Anisakidae Skrjabin \& Karokhin, 1945

Galeiceps longispiculum (Freitas \& Lent, 1941)

Pteronura brasiliensis (Gmelin), small intestine, stomach, Mato Grosso do Sul (Vicente et al. 1997) (CHIOC 12563-paratype, 15417-holotype and allotype, 15418 - 15419-paratypes, 18047, 18048).

\section{Superfamily Dioctophymatoidea Railliet, 1916}

\section{Family Dioctophymatidae (Railliet, 1915)}

Dioctophyma renale (Goeze, 1782)

Chrysocyon brachyurus (Illiger), kidney, abdominal cavity, stomach, Minas Gerais, São Paulo (Costa 
\& Freitas 1967; Costa \& Lima 1988; Vicente et al. 1997) (CHIOC 7699, 10080, 12440, 32962, 32963).

Galictis cuja (Molina), kidney, Rio de Janeiro (Barros et al. 1990) (CHIOC 731, 1347, 1441, 1578, 2044).

Galictis vittata (Schreber), kidney, Rio de Janeiro (Barros et al. 1990) (CHIOC 20868).

Lontra longicaudis (Olfers), kidney, abdominal cavity, stomach, unspecified locality (Barros et al. 1990; Vicente et al. 1997).

Nasua nasua (Linnaeus), kidney, abdominal cavity, stomach, unspecified locality (Barros et al. 1990; Vicente et al. 1997).

Speothos venaticus (Lund), unspecified site of infection, Mato Grosso (Barros et al. 1990) (CHIOC 169).

Dioctophyma sp.

Chrysocyon brachyurus (Illiger), abdominal cavity, São Paulo, NHR (CHIOC 21478).

Galictis vittata (Schreber), kidney, Rio de Janeiro, NHR (CHIOC 9785, 9997, 10013, 10119, 13746).

Nasua nasua (Linnaeus), abdominal cavity, Pará, NHR (CHIOC 10079).

Lontra longicaudis (Olfers), kidney, Rio de Janeiro, NHR (CHIOC 5443).

\section{Superfamily Dracunculoidea Cameron, 1934}

\section{Family Dracunculidae Leiper, 1912}

Dracunculus sp.

Lontra longicaudis (Olfers), under the skin, Rio de Janeiro (Noronha et al. 2002) (CHIOC 8124).

\section{Superfamily Filarioidea (Weinland, 1858)}

\section{Family Onchocercidae (Leiper, 1911)}

Dirofilaria incrassata (Molin, 1858)

Nasua nasua (Linnaeus), heart, under the skin, Mato Grosso, Mato Grosso do Sul, São Paulo (Vicente et al. 1997) (CHIOC 8507, 9545, 11611, 13441, 13442, 14489, 14483, 15104, 15271).

Procyon cancrivorus (G. Cuvier), under the skin, Rio de Janeiro, NHR (CHIOC 19829).

Nasua sp., heart, Mato Grosso, NHR (CHIOC 14483, 14489).

Dirofilaria repens Railliet \& Henry, 1911

Cerdocyon thous (Linnaeus), under the skin, Mato Grosso do Sul, NHR (CHIOC 15096, 15097, 15276, 15277).

Nasua nasua (Linnaeus), under the skin, heart, Mato Grosso do Sul (Noronha et al. 2002) (CHIOC $13215,13226,13454,13455,14979,15102,15103,15105,15107)$.

Dirofilaria sp.

Cerdocyon thous (Linnaeus), kidney, Mato Grosso do Sul (Noronha et al. 2002) (CHIOC 15096, 15097, 15276, 15277).

Galictis cuja (Molina), heart, Rio de Janeiro, NHR (CHIOC 2043).

Leopardus pardalis (Linnaeus), unspecified site of infection, Mato Grosso do Sul (Travassos 1945).

Lontra longicaudis (Olfers), under the skin, Rio de Janeiro (Noronha et al. 2002) (CHIOC 20103, 20104).

Nasua nasua (Linnaeus), pulmonary artery, heart, Mato Grosso do Sul, NHR (CHIOC 14980, 15016). Procyon cancrivorus (G. Cuvier), under the skin, Rio de Janeiro, NHR (CHIOC 8356).

Pteronura brasiliensis (Gmelin), under the skin, Mato Grosso do Sul, Rio de Janeiro, NHR (CHIOC 15415, 22160). 
Dirofilaria spectans Freitas \& Lent, 1949

Eira barbara (Linnaeus), heart, pulmonary artery, Mato Grosso do Sul (Noronha et al. 2002) (CHIOC $11612,18202,18203)$.

Lontra longicaudis (Olfers), heart, under the skin, Rio de Janeiro, Santa Catarina (Soto 2000; Noronha et al. 2002) (CHIOC 20108).

Pteronura brasiliensis (Gmelin), heart, pulmonary artery, Mato Grosso do Sul (Freitas \& Lent 1949;

Orihel 1965; Vicente et al. 1997) (CHIOC 13236-paratype, 17704-holotype and allotype, 17705paratype).

Dirofilaria striata (Molin, 1858)

Leopardus wiedii (Schinz), unspecified site of infection, unspecified locality (Lent \& Freitas 1937; Vicente et al. 1997).

Puma concolor (Linnaeus), unspecified site of infection, unspecified locality (Lent \& Freitas 1937; Vicente et al. 1997).

Family Filariidae (Cobbold, 1864)

Filaria carvalhoi Freitas \& Lent, 1937

Conepatus chinga (Molina), under the skin, Pernambuco (Freitas \& Lent 1937; Vicente et al. 1997) (CHIOC 9511—paratype, 9512-holotype and allotype).

Eira barbara (Linnaeus), under the skin, Mato Grosso do Sul, NHR (CHIOC 11613).

\section{Superfamily Gnathostomatoidea Railliet, 1895}

Family Gnathostomatidae Railliet, 1895

Gnathostoma americanum Travassos, 1925

Leopardus (Oncifelis) geoffroyi (D’Orbigny \& Gervais), stomach, unspecified locality (Vicente et al. 1997).

\section{Superfamily Heterakoidea Railliet \& Henry, 1914}

Family Lauroiidae Freitas, 1956

Lauroia heterospiculata (Gomes \& Pereira, 1970)

Leopardus wiedii (Schinz), large intestine, Amazonas (Vicente et al. 1997) (CHIOC 30527-holotype, 30528).

\section{Superfamily Metastrongyloidea Lane, 1917}

\section{Family Protostrongylidae Leiper, 1926}

Aelurostrongylus abstrusus (Railliet, 1898)

Puma (Herpailurus) yagouaroundi (Geoffroy), lung, Mato Grosso do Sul (Noronha et al. 2002) (CHIOC 15063).

Aelurostrongylus sp.

Cerdocyon thous (Linnaeus), heart, Mato Grosso do Sul, NHR (CHIOC 15161).

\section{Family Angiostrongylidae (Boerhm \& Gebauer, 1934)}

Angiostrongylus vasorum (Railliet, 1866)

Cerdocyon thous (Linnaeus), lung, heart, Minas Gerais (Duarte et al. 2007).

Lycalopex vetulus (Lund), lung, Minas Gerais (Lima et al. 1994).

Angiostrongylus raillieti (Travassos, 1927) 
Nasua nasua (Linnaeus), mesenterium, Paraná, NHR (CHIOC 33811).

Cerdocyon thous (Linnaeus), lung, right ventricle, pulmonary arteries, heart, Rio de Janeiro, Goiás

(Travassos 1927, Vicente et al. 1997) (CHIOC 4442, 5633, 5979).

Angiostrongylus sp.

Cerdocyon thous (Linnaeus), heart, lung, pulmonary artery, Mato Grosso do Sul, Rio de Janeiro (Travassos \& Freitas 1943) (CHIOC 9584).

Eira barbara (Linnaeus), heart, Mato Grosso do Sul, NHR (CHIOC 13035).

\section{Superfamily Physalopteroidea Sobolev, 1949}

\section{Family Physalopteridae (Railliet, 1893)}

Physaloptera anomala Molin, 1860

Panthera onca (Linnaeus), stomach, unspecified locality (Vicente et al. 1997).

Physaloptera digitata Schneider, 1866

Leopardus wiedii (Schinz), stomach, Pará (Noronha et al. 2002) (CHIOC 13505).

Puma concolor (Linnaeus), stomach, duodenum, Rio de Janeiro, São Paulo (Vicente et al. 1997)

(CHIOC 12279, 27275).

Physaloptera maxillaris Molin, 1860

Conepatus chinga (Molina), stomach, Pernambuco, Rio Grande do Sul (Vicente et al. 1997) (CHIOC

7731, 9958, 9959, 13976).

Physaloptera praeputialis Linstow, 1889

Cerdocyon thous (Linnaeus), stomach, Paraná, NHR (CHIOC 33809).

Chrysocyon brachyurus (Illiger), stomach, Minas Gerais (Costa \& Freitas 1967; Vicente et al. 1997).

Physaloptera semilanceolata Molin, 1860

Nasua nasua (Linnaeus), stomach, Mato Grosso do Sul, Pará (Vicente et al. 1997) (CHIOC 13499, 18930).

Physaloptera terdentata Molin, 1860

Puma concolor (Linnaeus), stomach, Rio de Janeiro (Vicente et al. 1997).

Physaloptera sp.

Cerdocyon thous (Linnaeus), stomach, Mato Grosso do Sul, NHR (CHIOC 15000, 15163).

Eira barbara (Linnaeus), stomach, Amazonas, NHR (CHIOC 21212).

Leopardus pardalis (Linnaeus), stomach, Amazonas, Mato Grosso do Sul, Pará (Travassos \& Freitas 1943; Vicente et al. 1997) (CHIOC 15032, 21199, 21262, 22773).

Leopardus wiedii (Schinz), small intestine, Pará, NHR (CHIOC 13493).

Nasua nasua (Linnaeus), small intestine, Mato Grosso, Mato Grosso do Sul, Rio de Janeiro (Travassos et al. 1927; Travassos \& Freitas 1943; Vicente et al. 1997) (CHIOC 29276).

\section{Superfamily Rhabditoidea Travassos, 1920}

\section{Family Strongyloididae Chitwood \& McIntosh, 1934}

Strongyloides sp.

Cerdocyon thous (Linnaeus), small intestine, Rio de Janeiro, NHR (CHIOC 5634).

Galictis vittata (Schreber), small intestine, Rio de Janeiro, NHR (CHIOC 10096).

\section{Superfamily Strongyloidea Weinland, 1858}

\section{Family Strongylidae Baird, 1853}


Strongylus sp.

Galictis vittata (Schreber), small intestine, Rio de Janeiro (Noronha et al. 2002).

\section{Family Syngamidae Leiper, 1912}

Mammomonogamus dispar (Diesing, 1851)

Puma concolor (Linnaeus), trachea, Rio de Janeiro (Vicente et al. 1997).

Mammomonogamus sp.

Puma concolor (Linnaeus), bronchi, Rio de Janeiro, NHR (CHIOC 8992).

\section{Superfamily Subuluroidea Travassos, 1930}

Family Subuluridae Yorke \& Maplestone, 1926

Subulura amazonica Pereira \& Machado Filho, 1968

Pteronura brasiliensis (Gmelin), small intestine, Amazonas, NHR (CHIOC 21205, 34213).

Subulura interrogans Lent \& Freitas, 1935

Pteronura brasiliensis (Gmelin), large intestine, Amazonas, NHR (CHIOC 21203).

\section{Superfamily Trichinelloidea Railliet, 1916}

\section{Family Trichuridae (Ransom, 1911)}

Calodium hepaticum (Brancoft, 1893)

Cerdocyon thous (Linnaeus), liver, Rio Grande do Sul (Ruas et al. 2003).

Chrysocyon brachyurus (Illiger), liver, unspecified locality (Vicente et al. 1997).

Lycalopex gymnocercus (G. Fischer), liver, Rio Grande do Sul (Ruas et al. 2003).

\section{Capillaria sp.}

Puma concolor (Linnaeus), urinary bladder, Rio de Janeiro, NHR (CHIOC 8571, 8572).

Pearsonema feliscati (Diesing, 1851)

Cerdocyon thous (Linnaeus), urinary bladder, Rio de Janeiro, NHR (CHIOC 13713).

Pearsonema linsi (Freitas \& Lent, 1935)

Cerdocyon thous (Linnaeus), urinary bladder, Rio de Janeiro, NHR (CHIOC 16103).

Galictis vittata (Schreber), urinary bladder, Rio de Janeiro (7849 - 7850 - holotype and allotype,

7851-paratype) (Vicente et al. 1997).

Pearsonema pearsoni Freitas \& Mendonça, 1960

Procyon cancrivorus (G. Cuvier), urinary bladder, Rio de Janeiro (Vicente et al. 1997) (CHIOC

27055-holotype and allotype).

Trichuris vulpis (Froelich, 1789)

Cerdocyon thous (Linnaeus), cecum, Mato Grosso do Sul, NHR (CHIOC 14958, 15087, 15157, 15158, 18924).

Trichuris sp.

Leopardus tigrinus (Schreber), unspecified site of infection, unspecified locality (Beldomenico et al. 2005).

Lycalopex gymnocercus (G. Fischer), cecum, Mato Grosso do Sul (Noronha et al. 2002) (CHIOC 13062).

Superfamily Trichostrongyloidea Cram, 1927

Family Molineidae Skrjabin \& Schulz, 1937 
Molineus barbaris Cameron, 1936

Eira barbara (Linnaeus), small intestine, Mato Grosso do Sul (Vicente et al. 1997) (CHIOC 14402). Molineus brachiurus Costa \& Freitas, 1967

Chrysocyon brachyurus (Illiger), small intestine, Minas Gerais (Costa \& Freitas 1967, Vicente et al. 1997).

Molineus felineus Cameron, 1923

Puma (Herpailurus) yagouaroundi (Geoffroy), small intestine, unspecified locality (Vicente et al. 1997).

Molineus major Cameron, 1936

Eira barbara (Linnaeus), small intestine, Mato Grosso do Sul, Rio de Janeiro (Vicente et al. 1997)

(CHIOC 14403 - 14409, 14377, 15335, 15678, 15679).

Pteronura brasiliensis (Gmelin), lung, Mato Grosso do Sul, NHR (CHIOC 12643).

Molineus nasuae Lent \& Freitas, 1938

Nasua nasua (Linnaeus), small intestine, Pará (Lent \& Freitas 1938b; Vicente et al. 1997) (CHIOC

9830 - holotype and allotype, 9844—paratype).

Molineus paraensis Travassos, 1937

Potos flavus (Schreber), small intestine, Pará (Lent \& Freitas 1938b; Vicente et al. 1997) (CHIOC

9754 - holotype and allotype, 9851).

Molineus sp.

Conepatus chinga (Molina), unspecified site of infection, Rio Grande do Sul (Noronha et al. 2002)

(CHIOC 14004, 14025).

Potos flavus (Schreber), intestine, Pará, NHR (CHIOC 9869).

Trichohelix tuberculata (Parona \& Stossich, 1901)

Conepatus chinga (Molina), small intestine, unspecified locality (Vicente et al. 1997).

\section{Phylum Platyhelminthes Gegenbaur, 1859}

\section{Class Cestoda Van Beneden, 1849}

Order Cyclophyllidea Van Beneden, 1850

Family Anoplocephalidae Choldkovsky, 1902

Atriotaenia sandgroundi (Baer, 1935)

Nasua nasua (Linnaeus), unspecified site of infection, unspecified locality (Yamaguti 1959; Travassos 1965)

\section{Family Mesocestoididae Fuhrmann, 1907}

Mesocestoides michaelseni Loemberg, 1896

Cerdocyon thous (Linnaeus), unspecified site of infection, unspecified locality (Yamaguti 1959; Travassos 1965).

\section{Family Taeniidae Ludwig, 1886}

Echinococcus oligarthrus (Diesing, 1863)

Puma concolor (Linnaeus), unspecified site of infection, unspecified locality (Yamaguti 1959; Travassos 1965; Schantz \& Colli 1973; Schmidt 1986).

Leopardus (Oncifelis) geoffroyi (D’Orbigny \& Gervais), unspecified site of infection, unspecified 
locality (Yamaguti 1959; Travassos 1965; Schmidt 1986).

Puma (Herpailurus) yagouaroundi (Geoffroy), unspecified site of infection, unspecified locality (Yamaguti 1959; Travassos 1965; Schmidt 1986).

Taenia crassipoda Rudolphi, 1819

Nasua nasua (Linnaeus), unspecified site of infection, unspecified locality (Yamaguti, 1959; Travassos 1965; Schmidt 1986).

Taenia macrocystis (Diesing, 1850)

Puma (Herpailurus) yagouaroundi (Geoffroy), unspecified site of infection, unspecified locality (Yamaguti 1959; Travassos 1965).

Leopardus (Oncifelis) geoffroyi (D’Orbigny \& Gervais), unspecified site of infection, unspecified locality (Yamaguti 1959; Travassos 1965).

Galictis sp. unspecified site of infection, unspecified locality (Yamaguti 1959; Travassos 1965).

Taenia omissa Luehe, 1910

Puma concolor (Linnaeus), unspecified site of infection, unspecified locality (Travassos 1965).

Leopardus (Oncifelis) geoffroyi (D’Orbigny \& Gervais), unspecified site of infection, unspecified locality (Travassos 1965).

Puma (Herpailurus) yagouaroundi (Geoffroy), unspecified site of infection, unspecified locality (Travassos 1965).

Taenia pisiformis Bloch, 1780

Leopardus (Oncifelis) geoffroyi (D’Orbigny \& Gervais), unspecified site of infection, unspecified locality (Travassos 1965).

Taenia taeniaeformis (Batsch, 1782)

Puma concolor (Linnaeus), unspecified site of infection, unspecified locality (Travassos 1965).

\section{Order Pseudophyllidea Carus, 1863}

\section{Family Diphyllobothriidae Lühe, 1910}

Diphyllobothrium gracilis (Baer, 1927)

Leopardus (Oncifelis) geoffroyi (D’Orbigny \& Gervais), unspecified site of infection, unspecified locality (Schmidt 1986).

Leopardus wiedii (Schinz), unspecified site of infection, unspecified locality (Yamaguti 1959; Travassos 1965; Schmidt 1986; Rego \& Schäffer 1992).

Puma (Herpailurus) yagouaroundi (Geoffroy), unspecified site of infection, unspecified locality (Schmidt 1986).

Diphyllobothrium latum (Linnaeus, 1758)

Leopardus (Oncifelis) geoffroyi (D’Orbigny \& Gervais), unspecified site of infection, unspecified locality (Travassos 1965).

Leopardus wiedii (Schinz), unspecified site of infection, unspecified locality (Travassos 1965).

Diphyllobothrium mansoni (Cobbold, 1883)

Cerdocyon thous (Linnaeus), small intestine, São Paulo (Santos et al. 2004).

Diphyllobothrium mansonoides Muller, 1935

Cerdocyon thous (Linnaeus), unspecified site of infection, unspecified locality (Schmidt 1986).

Diphyllobothrium trinitatis (Cameron, 1936)

Procyon cancrivorus (G. Cuvier), small intestine, São Paulo (Travassos 1965) (CHIOC 4469, 4473, 4474, 10975, 28802 - 28805).

Panthera onca (Linnaeus), intestine, Rio de Janeiro, NHR (CHIOC 28806). 


\section{Diphyllobothrium sp.}

Conepatus chinga (Molina), duodenum, São Paulo, Rio Grande do Sul, NHR (CHIOC 14013, 14014).

Cerdocyon thous (Linnaeus), small intestine, Rio de Janeiro, NHR (CHIOC 8295, 20867).

Lycalopex gymnocercus (G. Fischer), under the skin, Mato Grosso do Sul (Rego \& Schäffer 1992).

Panthera onca (Linnaeus), intestine, Rio de Janeiro, São Paulo (CHIOC 174, 8280, 30241, 30266);

faecal samples, Goiás (Carneiro et al. 1972).

Procyon cancrivorus (G. Cuvier), small intestine, Rio de Janeiro, NHR (CHIOC 30441).

Spirometra decipiens (Diesing, 1850)

Leopardus wiedii (Schinz), intestine, São Paulo, NHR (CHIOC 8270).

Panthera onca (Linnaeus), unspecified site of infection, São Paulo, NHR (CHIOC 4353, 4354).

Puma concolor (Linnaeus), intestine, unspecified locality (Škeř́́ková et al. 2006).

\section{Class Trematoda Rudolphi, 1808}

\section{Order Digenea Van Beneden, 1858}

\section{Family Dicrocoeliidae Looss, 1899}

Athesmia heterolecithodes (Braum, 1899)

Procyon cancrivorus (G. Cuvier), biliary duct, liver, Minas Gerais (Freitas 1962) (CHIOC 17503 17506, 17508, 17509).

Platynosomum illiciens (Braun, 1911)

Galictis vittata (Schreber), liver, biliary duct, Rio de Janeiro (Travassos 1944; Travassos et al., 1969) (CHIOC 10240, 10244, 17043, 17045).

Puma (Herpailurus) yagouaroundi (Geoffroy), liver, Pernambuco (Barbosa \& Pontual 1949; Rodrigues 1963).

Pseudathesmia paradoxa Travassos, 1942

Cerdocyon thous (Linnaeus), liver, biliary duct, Mato Grosso do Sul (Travassos 1942; Travassos 1944; Travassos et al. 1969) (CHIOC 13835 - 13839, 13843 - 13873, 13896 - 13902, 13923, 13926, 13927, 13941).

\section{Family Diplostomidae Poirier, 1886}

Alaria alata (Goeze, 1782)

Cerdocyon thous (Linnaeus), intestine, unspecified locality (Viana 1924; Travassos et al. 1969; Thatcher 1993).

Procyon cancrivorus (G. Cuvier), intestine, unspecified locality (Viana 1924).

Alaria clathrata Diesing, 1850

Pteronura brasiliensis (Gmelin), stomach, intestine, unspecified locality (Viana 1924; Thatcher 1993).

Alaria nattereri Lutz, 1933

Procyon cancrivorus (G. Cuvier), intestine, Minas Gerais (Lutz 1933) (CHIOC 17040, 17074 - 17080, 17273, 17275 - 17278, 17279, 17280 - 17289, 17291 - 17307, 17385, 24517, 24518, 24524, 24526).

Alaria pseudoclathrata (Krause, 1914)

Pteronura brasiliensis (Gmelin), intestine, unspecified locality (Yamaguti 1971; Thatcher 1993).

Diplostomum alarioides Dubois, 1937

Pteronura brasiliensis (Gmelin), large intestine, Mato Grosso do Sul (Travassos \& Freitas 1941;

Thatcher 1993) (CHIOC 12802, 15379). 
Family Echinostomatidae Looss, 1899

Baschkirovitrema incrassatum (Diesing, 1850)

Pteronura brasiliensis (Gmelin), intestine, unspecified locality (Viana 1924; Thatcher 1993).

Family Heterophyidae Odhner, 1914

Cryptocotyle thapari McIntosh, 1953

Pteronura brasiliensis (Gmelin), intestine, unspecified locality (Yamaguti 1971; Thatcher 1993)

Family Opisthorchiidae Braun, 1901

Amphimerus interruptus (Braum, 1901)

Speothos venaticus (Lund), biliary duct, Amazonas, NHR (CHIOC 1650).

Opisthorchis sp.

Puma (Herpailurus) yagouaroundi (Geoffroy), liver, Pará, NHR (CHIOC 21505 - 21507, 21516).

Family Paragonimidae Dollfus, 1939

Paragonimus rudis (Diesing, 1850)

Pteronura brasiliensis (Gmelin), lung, unspecified locality (Viana 1924; Yamaguti 1971).

Nasua sp., lung, unspecified locality (Thatcher 1993).

Family Schistosomatidae Stiles \& Hassal, 1898

Schistosoma mansoni Sambon, 1907

Procyon cancrivorus (G. Cuvier), unspecified site of infection, Minas Gerais (Coelho et al. 1976).

Family Strigeidae Railliet, 1919

Strigea vaginata (Brandes, 1888)

Galictis vittata (Schreber), musculature, Rio de Janeiro, NHR (CHIOC 17458, 25229, 25230 - 25232, 25249).

\section{Host-parasite list}

\section{Order Carnivora Bowdich}

\section{Suborder Caniformia Kretzoi}

\section{Family Canidae Fischer}

Cerdocyon thous

Aelurostrongylus sp. (NHR)

Alaria alata

Ancylostoma buckleyi

Ancylostoma caninum

Angiostrongylus raillieti

Angiostrongylus sp.

Angiostrongylus vasorum

Calodium hepaticum

Diphyllobothrium mansoni

Diphyllobothrium mansonoides 
Diphyllobothrium sp. (NHR)

Dirofilaria repens (NHR)

Dirofilaria sp.

Mesocestoides michaelseni

Oncicola sp. (NHR)

Pearsonema feliscati (NHR)

Pearsonema linsi (NHR)

Physaloptera praeputialis (NHR)

Physaloptera sp. (NHR)

Pseudathesmia paradoxa

Strongyloides sp. (NHR)

Toxocara canis

Trichuris vulpis (NHR)

Uncinaria carinii

Chrysocyon brachyurus

Ancylostoma caninum

Calodium hepaticum

Dioctophyma renale

Dioctophyma sp. (NHR)

Molineus brachiurus

Physaloptera preaputialis

Uncinaria sp.

Uncinaria stenocephala

Lycalopex gymnocercus

Ancylostoma caninum (NHR)

Calodium hepaticum

Diphyllobothrium sp.

Trichuris sp.

Lycalopex sp.

Ancylostoma caninum (NHR)

Lycalopex vetulus

Angiostrongylus vasorum

Speothos venaticus

Amphimerus interruptus (NHR)

Dioctophyma renale

\section{Family Mustelidae Fischer}

\section{Subfamily Lutrinae Bonaparte}

Lontra longicaudis

Dioctophyma renale

Dioctophyma sp. (NHR)

Dirofilaria sp.

Dirofilaria spectans

Dracunculus sp.

Lontra sp. 
Hexaglandula mutabilis (NHR)

Pteronura brasiliensis

Alaria clathrata

Alaria pseudoclathrata

Baschkirovitrema incrassatum

Cryptocotyle thapari

Diplostomum alarioides

Dirofilaria sp. (NHR)

Dirofilaria spectans

Galeiceps longispiculum

Molineus major (NHR)

Paragonimus rudis

Subulura amazonica (NHR)

Subulura interrogans (NHR)

\section{Subfamily Mustelinae Fischer}

Eira barbara

Angiostrongylus sp. (NHR)

Dirofilaria spectans

Filaria carvalhoi (NHR)

Molineus barbaris

Molineus major

Pachysentis gethi

Physaloptera sp.

Prosthenorchis elegans

Toxascaris leonina

Toxascaris sp. (NHR)

Galictis cuja

Dioctophyma renale

Dirofilaria sp. (NHR)

Pachysentis gethi (NHR)

Galictis sp.

Oncicola sigmoides

Taenia macrocystis

Galictis vittata

Dioctophyma renale

Dioctophyma sp. (NHR)

Pachysentis gethi (NHR)

Pearsonema linsi

Platynosomum illiciens

Strigea vaginata (NHR)

Strongyloides sp. (NHR)

Strongylus sp. 


\section{Family Mephitidae Bonaparte}

Conepatus chinga

Ancylostoma conepati (NGR)

Diphyllobothrium sp. (NHR)

Filaria carvalhoi

Molineus sp.

Neoncicola pintoi

Oncicola micracantha

Physaloptera maxillaris

Prosthenorchis elegans

Trichohelix tuberculata

Conepatus sp.

Oncicola sigmoides

\section{Family Procyonidae Gray}

Nasua nasua

Ancylostoma bidens

Angiostrongylus raillieti (NHR)

Atriotaenia sandgroundi

Dioctophyma renale

Dioctophyma sp. (NHR)

Dirofilaria incrassata

Dirofilaria repens

Dirofilaria sp. (NHR)

Molineus nasuae

Oncicola luehei

Physaloptera semilanceolata

Physaloptera sp.

Taenia crassipoda

Toxocara alienata

Toxocara canis (NHR)

Uncinaria bidens

Nasua sp.

Dirofilaria incrassata (NHR)

Paragonimus rudis

Potos flavus

Molineus paraensis

Molineus sp. (NHR)

Necator americanus (NHR)

Neoncicola potosi

Procyon cancrivorus

Alaria alata

Alaria nattereri

Ancylostoma bidens

Ancylostoma braziliense 
Athesmia heterolecithodes

Diphyllobothrium sp. (NHR)

Diphyllobothrium trinitatis

Dirofilaria incrassata (NHR)

Dirofilaria sp. (NHR)

Necator urichi

Pachysentis procyonis

Pearsonema pearsoni

Schistosoma mansoni

Skrjabinoclava thapari

Toxocara alienata

Toxocara canis (NHR)

Uncinaria bidens

Uncinaria maxillaris

\section{Suborder Feliformia Kretzoi}

\section{Family Felidae Fischer de Waldheim}

Leopardus pardalis

Ancylostoma braziliense

Dirofilaria sp.

Oligacanthorhynchus pardalis

Oncicola campanulata

Oncicola chibigouzouensis

Oncicola oncicola

Oncicola sp.

Physaloptera sp.

Prosthenorchis sp. (NHR)

Toxascaris leonina

Toxocara canis

Toxocara mistax (NHR)

Toxocara sp.

Leopardus tigrinus

Trichuris sp.

Leopardus wiedii

Ancylostoma pluridentatum (NGR)

Diphyllobothrium gracilis

Diphyllobothrium latum

Dirofilaria striata

Lauroia heterospiculata

Oligacanthorhynchus decrescens

Oncicola macrurae

Oncicola oncicola (NHR)

Physaloptera digitata

Physaloptera sp. (NHR)

Spirometra decipiens (NHR) 
Leopardus (Oncifelis) geoffroyi

Diphyllobothrium gracilis

Diphyllobothrium latum

Echinococcus oligarthrus

Gnathostoma americanum

Oligacanthorhynchus pardalis

Oncicola campanulata

Taenia macrocystis

Taenia omissa

Taenia pisiformis

Panthera onca

Ancylostoma caninum (NHR)

Ancylostoma pluridentatum (NGR)

Diphyllobothrium sp.

Diphyllobothrium trinitatis (NHR)

Oligacanthorhynchus pardalis

Oncicola campanulata

Oncicola oncicola

Physaloptera anomala

Spirometra decipiens (NHR)

Toxascaris leonina

Toxocara mistax

Puma concolor

Ancylostoma braziliense

Ancylostoma pluridentatum (NGR)

Capillaria sp. (NHR)

Dirofilaria striata

Echinococcus oligarthrus

Mammomonogamus dispar

Mammomonogamus sp. (NHR)

Oligacanthorhynchus pardalis

Oncicola campanulata

Oncicola magalhaesi

Physaloptera digitata

Physaloptera terdentata

Spirometra decipiens

Taenia omissa

Taenia taeniaeformis

Toxascaris leonina

Toxocara canis

Toxocara mistax

Puma (Herpailurus) yagouaroundi

Aelurostrongylus abstrusus

Ancylostoma bidens

Ancylostoma braziliense (NHR)

Ancylostoma caninum (NHR)

Diphyllobothrium gracilis 


\author{
Echinococcus oligarthrus \\ Molineus felineus \\ Oncicola oncicola \\ Oncicola paracampanulata \\ Opisthorchis sp. (NRH) \\ Platynosomum illiciens \\ Taenia macrocystis \\ Taenia omissa \\ Toxascaris leonina (NHR) \\ Toxocara mistax
}

\title{
Discussion
}

The literature about helminth parasites of carnivore mammals from Brazil are scarce and fragmented and not up to dated. The available information is mainly based on Travassos (1965), Travassos et al. (1969) and Vicente et al. (1997) that are not strictly compilations restricted to helminth of carnivores. According to Cheida et al. (2006) we have in Brazil a total of 29 species of wild carnivores mammals ( 21 of them referred on the present work), of which 16 species (55\%) referred on the List of Endangered Brazilian Species (Machado et al. 2005). Ten carnivore species reported in the present checklist (Chrysocyon brachyurus, Leopardus geoffroyi, L. pardalis, L. tigrinus, L. wiedii, Lontra longicaudis, Panthera onca, Pteronura brasiliensis, Puma concolor, Speothos venaticus) are considered endangered according to the former authors and IBGE (2006) and IUCN (2007), reinforcing the idea that parasites could extincted too along with its carnivores hosts.

\section{Acknowledgments}

To Sueli de Souza Lima (Universidade Federal de Juiz de Fora, Brazil) for provide some of the references cited. José L. Luque was supported by a research fellowship of the Conselho Nacional de Desenvolvimento Científico e Tecnológico (CNPq) of Brazil.

\section{References}

Amin, O.M. (1985) Classification. In: Crompton, D. W. T. \& Nickol, B. B. (Eds.). Biology of the Acanthocephala. Cambridge University Press, Cambridge, pp. 27-72.

Amin, O.M. (1987) Key to the families and subfamilies of Acanthocephala, with the erection of a new class (Polyacanthocephala) and a new order (Polyacanthorhynchida). Journal of Parasitology, 73, 1216-1219.

Anderson, R.C. \& Bain, O. (1976) Keys to the genera of the order Spirurida. Diplotriaenoidea, Aproctoidea and Filarioidea. In: Anderson, R. C., Chabaud, A. G. \& Willmott, S. (Eds.). CIH Keys to the nematode parasites of vertebrates 3. England, Commonwealth Agricultural Bureaux, Farnham Royal Bucks, pp. 59-116.

Anderson, R.C. \& Bain, O. (1982) Keys to genera of the superfamilies Rhabditoidea, Dioctophymatoidea, Trichinelloidea and Muspiceoidea. In: Anderson, R. C., Chabaud, A. G. \& Willmott, S. (Eds.). CIH Keys to the nematode parasites of vertebrates 9. England, Commonwealth Agricultural Bureaux, Farnham Royal Bucks, 26 pp.

Barbosa, F.A.S. \& Pontual, C. (1949) A new host for Platynosomum fastosum Kossack, 1910 (Trematoda, Dicrocoeliidae). Journal of Parasitology, 35, 546-547.

Barros, D.M., Lorini, M.L. \& Persson, V.G. (1990) Dioctophymosis in the Little Grison (Galictis cuja). Journal of Wildlife Diseases, 26, 538-539.

Beldomenico, P.M., Kinsella, J.M., Uhart, M.M., Gutierrez, G.L., Pereira, J., Ferreyra, H.D.V. \& Marull, C.A. (2005) Helminths of Geoffroy's cat, Oncifelis geoffroyi (Carnivora, Felidae) from the Monte desert, central Argentina. Acta 
Parasitologica, 50, 263-266.

Carneiro, J.R., Komma, M.D., Pereira, E. \& Santos, C.R. (1972) Nota sobre resultados coprológicos de felinos do Jardim Zoológico de Goiânia. Revista de Patologia Tropical, 1, 87-91.

Chabaud, A.G. (1975) Key to the genera of the order Spirurida. Part I. Camallanoidea, Dracunculoidea, Gnathostomatoidea, Physalopteroidea, Rictularoidea and Thelazoidea. In: Anderson, R. C., Chabaud, A. G. \& Willmott, S. (Eds.). CIH Keys to the nematode parasites of vertebrates 3. Part I. England, Commonwealth Agricultural Bureaux, Farnham Royal Bucks. 27 pp.

Chabaud, A.G. (1978) Key to the genera of superfamily Cosmocercoidea, Seuratoidea, Heterakoidea and Subuluroidea. In: Anderson, R. C., Chabaud, A. G. \& Willmott, S. (Eds.). CIH Keys to the nematode parasites of vertebrates 6. England, Commonwealth Agricultural Bureaux, Farnham Royal Bucks. 71 pp.

Cheida, C.C., Nakano-Oliveira, Fusco-Costa, R., Rocha-Mendes, F. \& Quadros, J. (2006) Ordem Carnivora. In: Reis, N. R., Peracchi, A. L., Pedro, W. A. \& Lima, I.P. (Eds.). Mamíferos do Brasil. Universidade Estadual de Londrina, Londrina, pp. 231-275.

Coelho, P.M.Z., Dias, M., Mayrink, W., Magalhaes, P.A., Melo, M.N. \& Costa, C.A. (1976) Crab-eating Raccoon, Procyon cancrivorus nigripes (Mivart 1885) (Carnivora: Procyonidae) Naturally Infected with Schistosoma mansoni in Minas Gerais State, Brazil. Journal of Parasitology, 62, 748.

Costa, H.M.A. \& Freitas, M.G. (1967) Alguns helmintos parasitos do guará (Chrysocyon brachiurus (Illiger)) com a descrição de Molineus brachiurus n. sp. (Nematoda, Trichostrongylidae). Arquivos da Escola de Veterinária, $U F M G, 19,25-29$.

Costa, H.M.A. \& Lima, W.S. (1988) Dioctophyme renale (Goeze, 1782): ocorrências em Minas Gerais. Arquivo Brasileiro de Medicina Veterinária e Zootecnia, 40, 243-244.

Costa, L.P., Leite, Y.L., Mendes, S.L. \& Ditchfield, A.D. (2005) Mammal Conservation in Brazil. Conservation Biology, 19, 672-679.

Duarte, F.H., Vieira, F.M., Louzada, G.L., Bessa, E.C.A. \& Souza Lima, S. (2007) Occurrence Angiostrongylus vasorum (Raillet, 1866) (Nematoda, Angiostrongylidae) in Cerdocyon thous Linnaeus, 1766 (Carnivora, Canidae) in Minas Gerais State, Brazil. Arquivo Brasileiro de Medicina Veterinária e Zootecnia, 59, 1086-1088.

Durette-Desset, M.C. (1983) Keys to the genera of superfamily Trichostrongyloidea. In: Anderson, R. C., Chabaud, A. G. \& Willmott, S. (Eds.). CIH Keys to the nematode parasites of vertebrates 10. England, Commonwealth Agricultural Bureaux, Farnham Royal Bucks. 86 pp.

Freitas, J.F.T. \& Lent, H. (1937) Segunda especie do genero Filaria Mueller, 1787, s. str. Memórias do Instituto Oswaldo Cruz, 32, 423-426.

Freitas, J.F.T. \& Lent, H. (1949) Nova “Dirofilaria” parasita do aparelho circulatório de ariranha (Nematoda, Filarioidea). Revista Brasileira de Biologia, 9, 377-380.

Freitas, J.F.T. (1962) Notas sobre o gênero Athesmia Looss, 1899. Arquivos do Museu Nacional, Rio de Janeiro, 52, 85104.

Gardner, S.L. \& Campbell, M.L. (1992) Parasites as probes for biodiversity. Journal of Parasitology, 78, 596-600.

Gibson, D.I., Jones, A. \& Bray, R.A. (Eds.). (2002) Keys to the Trematoda, Volume 1. CABI Publishing, London. 544p.

IBGE. (2006) Mapa da fauna ameaçada de extinção - Mamíferos, Répteis e Anfíbios 2006. Avaliable from ftp:// geoftp.ibge.gov.br/mapas/tematicos/mapas_murais/mamiferos_2006.pdf. (accessed 04 October 2007).

IUCN - International Union for Conservation of Nature and Natural Resources. (2007) 2007 IUCN Red List of Threatened Species. Avaliable from http://www.iucnredlist.org (accessed 04 October 2007).

Jones, A., Bray, R.A. \& Gibson, D.I. (Eds.). (2005) Keys to the Trematoda, Volume 2. CABI Publishing, London, 768 pp.

Khalil, L.F., Jones, A. \& Bray, R.A. (Eds.). (1994) Key to the cestodes of vertebrates. Wallingford: CAB International, $751 \mathrm{pp}$.

Lent, H. \& Freitas, J.F.T. (1937) Contribuição ao estudo do gênero Dirofilaria Railliet \& Henry, 1911. Memórias do Instituto Oswaldo Cruz, 32, 37-54.

Lent, H. \& Freitas, J.F.T. (1938a) Pesquisas helminthologicas realisadas no estado do Pará. VI. Acanthocephala. Memórias do Instituto Oswaldo Cruz, 33, 455-459.

Lent, H. \& Freitas, J.F.T. (1938b) Pesquisas helminthologicas realisadas no estado do Pará. IV. Trichostrongylideos de mammiferos. Memórias do Instituto Oswaldo Cruz, 33, 363-380.

Lewinsohn, T.M. \& Prado P. I. (2002) Biodiversidade brasileira: síntese do estado atual de conhecimento. Editora Contexto, São Paulo. 176p.

Lichtenfels, J.R. (1980a) Keys to the genera of the superfamily Strongyloidea. In: Anderson, R. C., Chabaud, A. G. \& Willmott, S. (Eds.). CIH Keys to the nematode parasites of vertebrates 7. England, Commonwealth Agricultural Bureaux, Farnham Royal Bucks. 41p.

Lichtenfels, J.R. (1980b) Keys to the genera of the superfamilies Ancylostomatoidea and Diaphanocephaloidea. In: Anderson, R. C., Chabaud, A. G. \& Willmott, S. (Eds.). CIH Keys to the nematode parasites of vertebrates 8. England, Commonwealth Agricultural Bureaux, Farnham Royal Bucks. 26 pp.

Lima, W.S., Guimarães, M.P. \& Lemos, I.S. (1994) Occurrence of Angiostrongylus vasorum in the lungs of the Brazilian 
fox Dusicyon vetulus. Journal of Helminthology, 68, 87.

Lutz, A. (1933) Considerações sobre o Distomum tetracystis Gastaldi e formas semelhantes, erroneamente chamadas Agamodistomos. Memórias do Instituto Oswaldo Cruz, 27, 33-49.

Machado Filho, D.A. (1940) Pesquisas helmintológicas realizadas no estado de Mato Grosso-Acanthocephala. Memórias do Instituto Oswaldo Cruz, 35, 593-601.

Machado Filho, D.A. (1950) Revisão do gênero Prosthenorchis Travassos, 1915 (Acanthocephala). Memórias do Instituto Oswaldo Cruz, 48, 495-544.

Machado Filho, D.A. (1962) Nova espécie do gênero "Oncicola" Travassos, 1916 (Metacanthocephala, Archiacanthocephala, Pachysentidae). Revista Brasileira de Biologia, 22, 367-369.

Machado Filho, D.A. (1963a) “Oncicola chibigouzouensis” sp. n. parasito de “Felis chibigouzou” (Griff.) (Archiacanthocephala, Pachysentidae). Revista Brasileira de Biologia, 23, 15-17.

Machado Filho, D.A. (1963b) “Oncicola paracampanulata” sp. n. parasito de "Herpailurus jaguarundi" (Fisch.) (Archiacanthocephala, Pachysentidae). Revista Brasileira de Biologia, 23, 153-155.

Machado Filho, D.A. (1964) Contribuição para o conhecimento do gênero "Oncicola" Travassos, 1916 (Archiacanthocephala, Pachysentidae). Revista Brasileira de Biologia, 24, 23-30.

Machado, A.B.M., Martins, C.S. \& Drummond, G.M. (2005) Lista da fauna brasileira ameaçada de extinção: incluindo as espécies quase ameaçadas e deficientes em dados. Fundação Biodiversitas, Belo Horizonte, 158 pp.

Marteu, M. (1977) Oncicola venezuelensis n. sp. (Archiacanthocephala; Oligacanthorhynchida), parasite de l'Ocelot (Felis pardalis L.). Annales de Parasitologie, 52, 25-33.

Meyer, A. (1931) Neue Acanthocephalen aus dem Berliner Museum. Begriindung eines neuen Acanthocephalensystems auf Grund einer Untersuchung der Berliner Sammlung. Zoologische Jahrbücher. Abteilung für Systematik, Okologie und Geographie der Tiere, 62, 53-108.

Mundim, M.J.S., Machado, M.I., Bevilaqua, E., Mundim, A.V., Maywald, P.G. \& Oliveira, M.G. (1991) Ocorrência e identificação de Ancilostomatídeos em lobo-guará (Chrysocyon brachyurus, Illiger, 1811) da região do Triangulo Mineiro, Minas Gerais, Brazil. Brazilian Journal of Veterinary Research and Animal Science, 28, 39-43.

Noronha, D., Bragança, R., Vicente, J.J. \& Muniz Pereira, L.C. (2004) Coleções particulares incorporadas à Coleção Helmintológica do Instituto Oswaldo Cruz (CHIOC). I: Coleção do Instituto Pasteur de São Paulo. Revista Brasileira de Zoologia, 21, 303-305.

Noronha, D., Vicente, J.J. \& Pinto, R.M. (2002) A survey of new records for nematodes from mammals deposited in the Helminthological collection of the Institute Oswaldo Cruz (CHIOC). Revista Brasileira de Zoologia, 19, 945-949.

Orihel, T.C. (1965) Dirofilaria lutrae sp. n. (Nematoda: Filarioidea) from Otters in the Southeast United States. Journal of Parasitology, 51, 409-413.

Poulin, R. \& Morand, S. (2004) Parasite biodiversity. Smithsonian Books, Washington D. C., 216 pp.

Rego, A.A. \& Schäffer, G.V. (1992) Esparganose em alguns vertebrados do Brasil. Dificuldades na indentificação das espécies de Lueheella (Spirometra). Memórias do Instituto Oswaldo Cruz, 87, 213-216.

Rodrigues, H.O. (1963) Contribuição ao estudo do gênero Platynosomum Looss, 1907 (Trematoda, Dicrocoeliidae). Memórias do Instituto Oswaldo Cruz, 61, 507-515.

Ruas, J.L., Soares, M.P., Farias, N.A.R. \& Brum, J.G.W. (2003) Infecção por Capillaria hepatica em carnívoros silvestres (Lycalopes gymnocercus e Cerdocyon thous) na região sul do Rio Grande do Sul. Arquivos do Instituto Biológico, 70, 147-150.

Santos, K.R., Catenacci, L.S., Pestelli, M.M., Takahira, R.K., Lopes, R.S. \& Silva, R.J. (2003) First report of Ancylostoma buckleyi Le Roux and Biocca, 1957 (Nematoda: Ancylostomatidae) infecting Cerdocyon thous Linnaeus, 1766 (Mammalia: Canidae) from Brazil. Revista Brasileira de Parasitologia Veterinária, 12, 179-181.

Santos, K.R., Catenacci, L.S., Pestelli, M.M., Takahira, R.K. \& Silva, R.J. (2004) First report of Diphyllobothrium mansoni (Cestoda, Diphyllobothridae) infecting Cerdocyon thous (Mammalia, Canidae) in Brazil. Arquivo Brasileiro de Medicina Veterinária e Zootecnia, 56, 796-798.

Schantz, P.M. \& Colli, C. (1973) Echinococcus oligarthrus (Diesing, 1863) from Geoffroy's cat (Felis geoffroyi D’Orbigny y Gervais) in temperate South America. Journal of Parasitology, 59, 1138-1140.

Schmidt, G.D. (1972) Revision of the Class Archiacanthocephala Meyer, 1931 (Phylum Acanthocephala), with Emphasis on Oligacanthorhynchidae Southwell et Macfie, 1925. Journal of Parasitology, 58, 290-297.

Schmidt, G.D. (1986) Handbook of tapeworm identification. CRC Press, Florida, 75 pp.

Škeř́ková, A., Brabec, J., Kuchta, R., Jiménez, J.A., García, H.H. \& Scholz, T. (2006) Is the human-infecting Diphyllobothrium pacificum a valid species or just a South American population on the holartic fish broad tapworm, $D$. latum? American Journal of Tropical Medicine and Hygiene, 75, 307-310.

Soto, J.M.R. (2000) First record of Dirofilaria spectans Freitas \& Lent, 1949 (Nematoda, Filariidae) in Lutra longicaudis Olfers, 1818 (Mammalia, Mustelidae). Revista Brasileira de Parasitologia Veterinária, 9, 157-158.

Thatcher, V.E. (1993) Trematódeos Neotropicais. Instituto Nacional de Pesquisa da Amazônia, Manaus, 553 pp.

Travassos, L \& Freitas, J.F.T. (1943) Relatório da sétima excursão científica do Instituto Oswaldo Cruz, realizada a zona 
da Estrada de Ferro Noroeste do Brasil, em maio de 1942. Memórias do Instituto Oswaldo Cruz, 38, $385-412$.

Travassos, L. \& Freitas, J.F.T. (1941) II-Pesquisas parasitológicas. Memórias do Instituto Oswaldo Cruz, 36, $272-295$.

Travassos, L. (1915) Uncinaria carinii n. sp. (nota prévia). Brazil Médico, 29, 79-80.

Travassos, L. (1917) Contribuições para o conhecimento da fauna helmintológica brasileira: VI Revisão dos acantocéfalos brasileiros, parte I Giganthorhynchidae Hamann, 1892. Memórias do Instituto Oswaldo Cruz, 9, 5-62.

Travassos, L. (1927) Novos nematódeos. Boletin Biológico, 6, 52-61.

Travassos, L. (1942) Novo Dicrocoeliidae parasito de carnívoros: Pseudathesmia paradoxa n. g., n. sp., com uma nota sôbre o gênero Athesmia Looss, 1899. Revista Brasileira de Biologia, 2, 349-357.

Travassos, L. (1944) Revisão da família Dicrocoeliidae Odner, 1910. Monografias do Instituto Oswaldo Cruz, 2, 1 -357.

Travassos, L. (1945) Relatório da excursäo do Instituto Oswaldo Cruz ao Rio Paraná (Porto Cabral), em marco e abril de 1944. Memórias do Instituto Oswaldo Cruz, 42, 151-165.

Travassos, L. (1965) Contribuição para o Inventário Crítico da Zoologia no Brasil. Fauna Helmintológica: Considerações Preliminares - Cestódeos. Publicações Avulsas do Museu Nacional, Rio de Janeiro, 84 p.

Travassos, L., Freitas, J.F.T. \& Kohn, A. (1969) Trematódeos do Brasil. Memórias do Instituto Oswaldo Cruz, 67, 1-886.

Travassos, L., Pinto, C. \& Muniz, J. (1927) Excursão scientifica ao Estado de Matto Grosso na Zona do Pantanal (margens dos rios S. Lourenço e Cuyabá) realizada em 1922. Memórias do Instituto Oswaldo Cruz, 20, 249-269.

Viana, L. (1924) Tentativa de catalogaçäo das espécies brazileiras de trematodeos. Memórias do Instituto Oswaldo Cruz, 17, 95-227.

Vicente, J.J., Rodrigues, H.O., Gomes, D.C. \& Pinto, R.M. (1997) Nematóides do Brasil. Parte V: Nematóides de mamíferos. Revista Brasileira de Zoologia, 14, 1-452.

Wozencraft, W. (2005) Order Carnivora. In: Wilson, D. E. \& Reeder, D. M. (Eds.) Mammal Species of the World: A taxonomic and geographic reference. The Johns Hopkins University Press, Baltimore and London, pp. 532-628

Yamaguti, S. (1959) Systema Helminthum. Vol II. The Cestodes of Vertebrates. Interscience Publishers, New York. 860 pp.

Yamaguti, S. (1963) Systema Helminthum. Vol. V. The Acanthocephala of Vertebrates. Interscience Publishers, New York, $860 \mathrm{pp}$.

Yamaguti, S. (1971) Synopsis of the digenetic trematodes of vertebrates. Keigaku Publishing Company, Tokyo, 1074 pp. 